\title{
Patient-specific prediction of long-term outcomes will change stroke rehabilitation for the better
}

India Walford ${ }^{1}$, Jane Rondina², Nick S Ward ${ }^{1,3}$

${ }^{1}$ Department of Clinical and Motor Neuroscience, UCL Queen Square Institute of Neurology, London, UK.

${ }^{2}$ Department of Brain Repair and Rehabilitation, UCL Queen Square Institute of Neurology, London, UK.

${ }^{3}$ The National Hospital for Neurology and Neurosurgery, Queen Square, London, UK

Stroke is the commonest cause of neurological disability and yet our ability to predict long-term outcomes remains poor. The paper by Selles et a ${ }^{1}$ used upper limb outcomes from 450 patients with first-time ischaemic stroke to take a refreshingly different approach to the prediction problem. Firstly, allowing repeated clinical measures to contribute to the prediction acknowledges what most clinicians already know - that rate of clinical change is helpful in prognostication. Secondly, rather than predict outcome at a single future time point, they have created likely recovery trajectories (with confidence intervals) for individual patients. Thirdly, access to this predictive model is freely available online so that stroke services around the world can more accurately begin to make predictions of individual recovery. Although the current approach concerns upper limb recovery, the principle should apply to all domains.

Current methodological approaches to prediction rely on linear and logistical regression models requiring a single measurement at a pre-determined time post-stroke to predict performance at a specific future time point. Regression models are by nature inflexible because they yield temporally fixed predictions from temporally fixed input data. Selles et $a l^{1}$ however, recognised that using nontemporally bound repeated measurements might be more accurate and clinically useful. To achieve this, they used a mixed-effects model to account for correlations between repeated measures within patients, explicitly avoiding the problematic mathematical coupling when using a baseline score to predict a change in the same score. Not surprisingly, predictions became increasingly accurate as more data became available.

Clinicians are often uncomfortable with making predictions on the basis that 'it might be wrong'. The consequence is clinical uncertainty, misinformation and a rather nihilistic view about the potential for recovery based on a desire to avoid providing 'false hope'. The inclusion of confidence intervals here is crucial to illustrate a range of possible data-led outcomes and move us away from the dichotomy of 'you will/won't walk again' statements. It is also important to say that making a domain-specific prediction at the level of impairment does not tell us what is possible at the level of activity or 
participation, or what is possible in other domains. It is always possible to help patients in some way and so worries that patients would be 'written off' and treatment withheld should not deter us from striving to make better predictions of long-term outcome.

We can also look at predictions as challenges to be overcome. Any predictive model in stroke recovery is only as good as the treatment provided (in the case of Selles et al ${ }^{1}$ patients received standard rehabilitation treatment according to the Dutch rehabilitation guidelines). It follows that predictive models could be the best way to evaluate novel treatments both at individual or group level. Rather than look for minimum clinically important differences in outcome scores, we should ask whether treatment, either in the early or chronic phase ${ }^{2,3}$, led to the prediction being significantly outperformed. As well as being useful in clinical trials, this approach could also be used to benchmark local and national services.

The final key reason that predictive models are important is that they will allow stratification in clinical trials based on expected outcomes. For upper limb recovery, this is particularly important for patients that present with a more severe impairment where early predictions about late outcomes are notoriously inaccurate. If clinical trials of prospective treatments could target homogenous groups of patients with similar expected outcomes, then clinical effects would be easier to detect, sample sizes would be smaller and trials quicker to conduct ${ }^{4}$.

Selles et $a l^{1}$ used only clinical scores in their model. Empirical questions remain concerning whether neuroimaging or neurophysiological measures could improve predictions ${ }^{5,6}$. Nevertheless, developing predictive models of long-term post-stroke outcomes is low hanging fruit and must be a priority for research funding. Creating accurate predictive models across motor, language and cognitive domains would surely transform evaluation and research in stroke recovery.

\section{References}

1. Selles, R. W. et al. Computerised patient-specific prediction of the recovery profile of upper limb capacity within stroke services: the next step. J. Neurol. Neurosurg. Psychiatry (2021) doi:10.1136/jnnp-2020-324637.

2. Ward, N. S., Brander, F. \& Kelly, K. Intensive upper limb neurorehabilitation in chronic stroke: outcomes from the Queen Square programme. J. Neurol. Neurosurg. Psychiatry 90, 498-506 (2019). 
3. Ganesh, A., Gutnikov, S. A., Rothwell, P. M. \& Oxford Vascular Study. Late functional improvement after lacunar stroke: a population-based study. J. Neurol. Neurosurg. Psychiatry (2018) doi:10.1136/jnnp-2018-318434.

4. Winters, C., Heymans, M. W., van Wegen, E. E. H. \& Kwakkel, G. How to design clinical rehabilitation trials for the upper paretic limb early post stroke? Trials 17, 468 (2016).

5. Smith, M.-C., Ackerley, S. J., Barber, P. A., Byblow, W. D. \& Stinear, C. M. PREP2 Algorithm Predictions Are Correct at 2 Years Poststroke for Most Patients. Neurorehabil. Neural Repair 33, 635-642 (2019).

6. Hope, T. M. H., Seghier, M. L., Leff, A. P. \& Price, C. J. Predicting outcome and recovery after stroke with lesions extracted from MRI images. Neurolmage Clin. 2, 424-433 (2013). 